\title{
IDENTIFICATION OF IMAGE SPAM BY USING LOW LEVEL \& METADATA FEATURES
}

\author{
Anand Gupta $^{1}$, Chhavi Singhal ${ }^{2}$ and Somya Aggarwal ${ }^{1}$ \\ ${ }^{1}$ Department of Computer Engineering, \\ ${ }^{2}$ Department of Electronic and Communication Engineering \\ Netaji Subhas Institute of Technology, New Delhi, India \\ \{omaranand, chhavisinghal28, somya3322\}@gmail.com
}

\begin{abstract}
Spammers are constantly evolving new spam technologies, the latest of which is image spam. Till now research in spam image identification has been addressed by considering properties like colour, size, compressibility, entropy, content etc. However, we feel the methods of identification so evolved have certain limitations due to embedded obfuscation like complex backgrounds, compression artifacts and wide variety of fonts and formats. To overcome these limitations, we have proposed 2 methodologies(however there can be more). Each methodology has 4 stages. Both the methodologies are almost similar except in the second stage where methodology I extracts low level features while the other extracts metadata features. Also a comparison between both the methodologies is shown. The method works on images with and without noise separately. Colour properties of the images are altered so that OCR (Optical Character Recognition) can easily read the text embedded in the image. The proposed methods are tested on a dataset of 1984 spam images and are found to be effective in identifying all types of spam images having (1) only text, (2) only images or (3) both text and images. The encouraging experimental results show that the methodology I achieves an accuracy of $92 \%$ while the other achieves an accuracy of $93.3 \%$.
\end{abstract}

\section{KEYWORDS}

Low level feature, anti obfuscation technique, noise \& entropy

\section{INTRODUCTION}

Image spam is a kind of spam in e-mail where the message text of the spam is presented as an image file. Anti spam filters label an e-mail (with image attached) as spam if they find suspicious text embedded in that image. For that, the filters employ OCR that reads text embedded in images. It works by measuring the geometry in images, searching for shapes that match the shapes of letters, then translating a matched geometric shape into real text. To defeat OCR, spammers upset the geometry of letters enough-by altering colours, for example - so that OCR can't "see" a letter, even though the human eye easily recognize it. To overcome this falsity, low level and metadata features of images are extracted as they are effective against randomly added noises and simple translational shift of the images. We now review the prior significant work in the area of image spam identification.

\section{PRIOR WORK}

Till now spam identification has been carried out by considering the following spam image properties.

1. Content (C) 2. Metadata features (M) 3. Low level features (L) 4. Text region (T)

DOI : $10.5121 /$ ijnsa.2012.4213 
International Journal of Network Security \& Its Applications (IJNSA), Vol.4, No.2, March 2012

The following matrix shows the properties as used in the previous works. Whereas the left most column shows the reference numbers, the top most row shows the properties employed as given above. Numerals ' 1 ' and ' 0 ' mean that the given property is used and not used respectively.

\begin{tabular}{|c|c|c|c|c|}
\hline & C & $\mathbf{M}$ & $\mathbf{L}$ & $\mathbf{T}$ \\
\hline [1] & 1 & 0 & 0 & 0 \\
\hline [2] & 1 & 0 & 0 & 0 \\
\hline [3] & 0 & 1 & $\mathbf{0}$ & $\mathbf{0}$ \\
\hline [4] & 0 & $\mathbf{0}$ & $\mathbf{1}$ & $\mathbf{0}$ \\
\hline [5] & 0 & 1 & $\mathbf{1}$ & 0 \\
\hline [6] & 1 & $\mathbf{1}$ & $\mathbf{1}$ & 0 \\
\hline [7] & 0 & 0 & 0 & $\mathbf{1}$ \\
\hline
\end{tabular}

In [1], a scheme is proposed which implements a spam filter based on the text in the subject and body fields of e-mails, and the text embedded into attached images. The conventional document processing steps (tokenization, indexing and classification) are improved upon in [1] by employing text extraction using OCR from attached images. SpamAssassin (SA)[2] is a widely deployed filter program that uses OCR software to pull words out of images and then uses the text based traditional methods to filter spam. This happens to be an improvement of the earlier.

In [3][13], a spam filtering technique has been proposed that uses image information (metadata features) such as file size, area, compressibility etc., and states a characteristics that appears for each information entity.

On the contrary, [4] identifies spam using a probabilistic boosting tree based on global image features (low level features), i.e. colour and gradient orientation histograms. In the year 2010, a feature extraction scheme that concentrates on both low-level and metadata features is proposed in [5].It does not rely on extracting the text.

In [6], a mechanism is proposed to ascertain spam embedded main body e-mail file, called as Partial Image Spam Inspector (PIMSI). The significant feature of this method is that it evaluates both low level features and metadata features to confirm whether a mail is spam or not. It analyses spam images by dividing it into 2 databases. Database of object image spam consists of images and its properties such as RGB colours, contrast and brightness. In the database of Vocal Spam, all keywords of the advertised spam images are recorded and are compared with the text extracted using OCR.

To overcome the shortcomings of the methods mentioned above, a spam identification model has been proposed in [7] which does not exploit low level features and OCR to extract text from images. Instead, it identifies spam by using the visual-BOW (VBOW) based duplicate image detection and statistical language model. Computation-efficient edge-detection method is used to locate possible text regions, and then text coverage rate in an image is calculated. Text region in a large majority of normal image is less than $15 \%$, while text region in most spam is larger than such a threshold.

\subsection{Motivation}

The following drawbacks in prior related works have motivated us to develop a method that mitigates them.

Nowadays, spammers use different image processing technologies to vary the properties of individual messages e.g. by changing the foreground colours, backgrounds, font types or even rotating and adding artifacts to the images. Thus, they pose great challenges to conventional spam filters. 
International Journal of Network Security \& Its Applications (IJNSA), Vol.4, No.2, March 2012

[4][6][5][8] use colour histograms to distinguish spam images from normal images. Colour histograms of natural images tend to be continuous, while the colour histograms of artificial spam images tend to have some isolated peaks. We point out however that the discriminating capability of the above feature is not likely to be satisfactory, since colour distribution is solely dependent on the format of the image. Figure 1 shows a sample image and the difference between its colour histograms when saved with different formats (jpeg and gif) is illustrated in Figures 2 and 3.

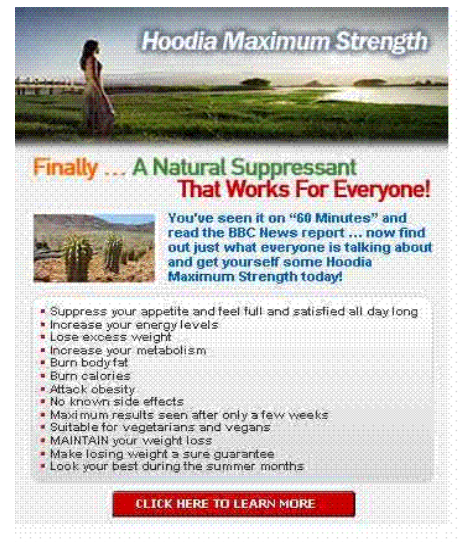

Figure 1. Original Image

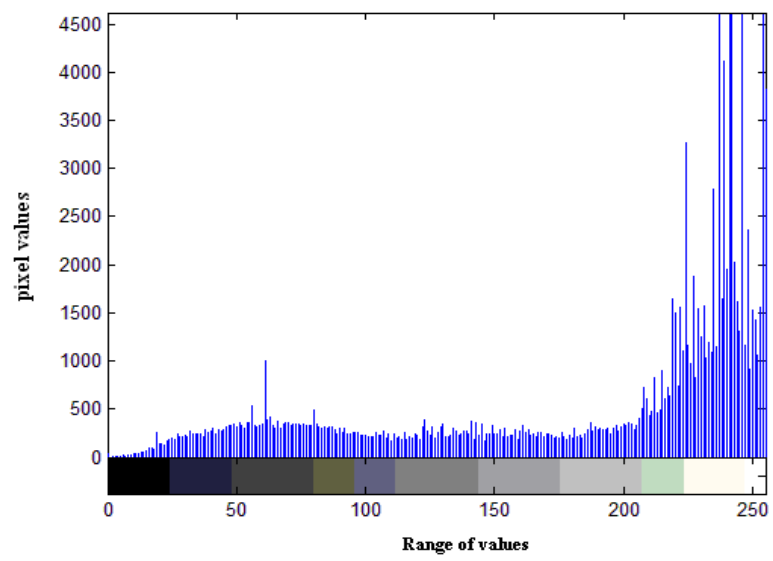

Figure 2. Colour histogram of fig 1 in jpeg format 


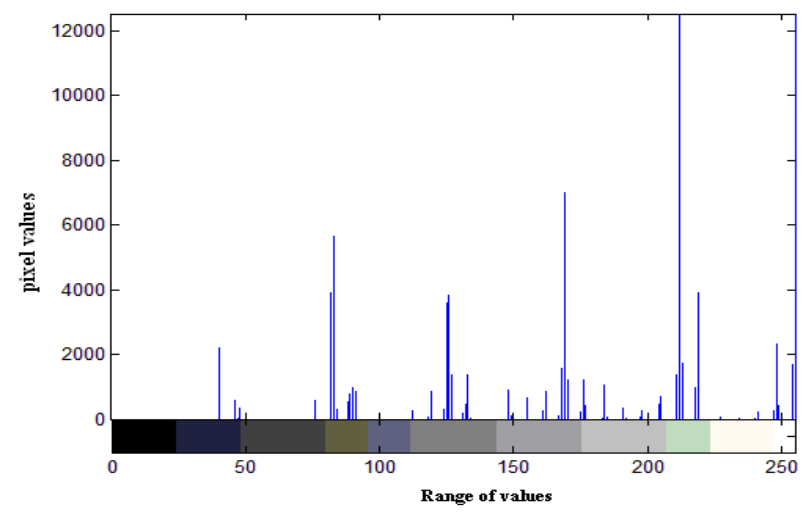

Figure 3. Colour histogram of fig 1 in gif format

[3][5][6] use metadata features. Different images can have similar (even same) metadata features. This technology of image spam detection may be wrong and has low accuracy rate. After carrying out experiments we have found that $58.65 \%$ of spam images and $44.28 \%$ of normal images are smaller than 10KB. It implies that metadata features can be similar for both kind of images. Hence it is not a reliable method to distinguish between spam images from normal images.

The method mentioned in [7] has helped achieve significant results in identifying spam images which contain only text. However, few spam images contain both text and images. Figures 4 and 5 show that edge detection is not able to distinguish between text and images. Also edge detection will detect noise and treat it as text.

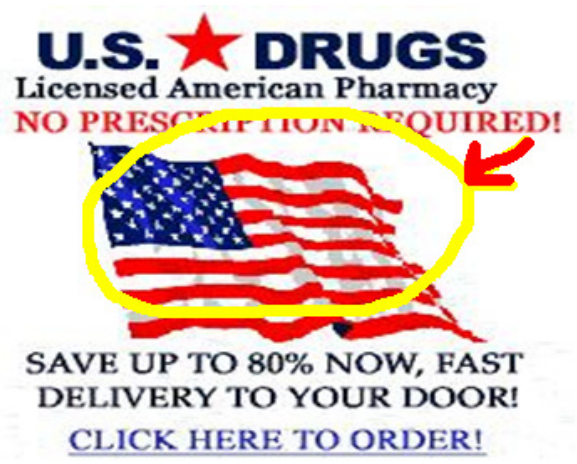

Figure 4. Original Image

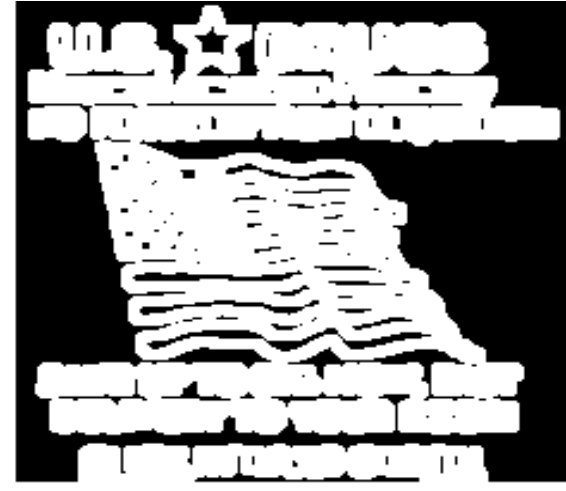

Figure 5. Edge detection of fig 4

\section{SYSTEM ARCHITECTURE}

We have proposed two methodologies based on low level features and entropy. Figure 6 depicts the basic framework of the two methodologies employed. 
International Journal of Network Security \& Its Applications (IJNSA), Vol.4, No.2, March 2012

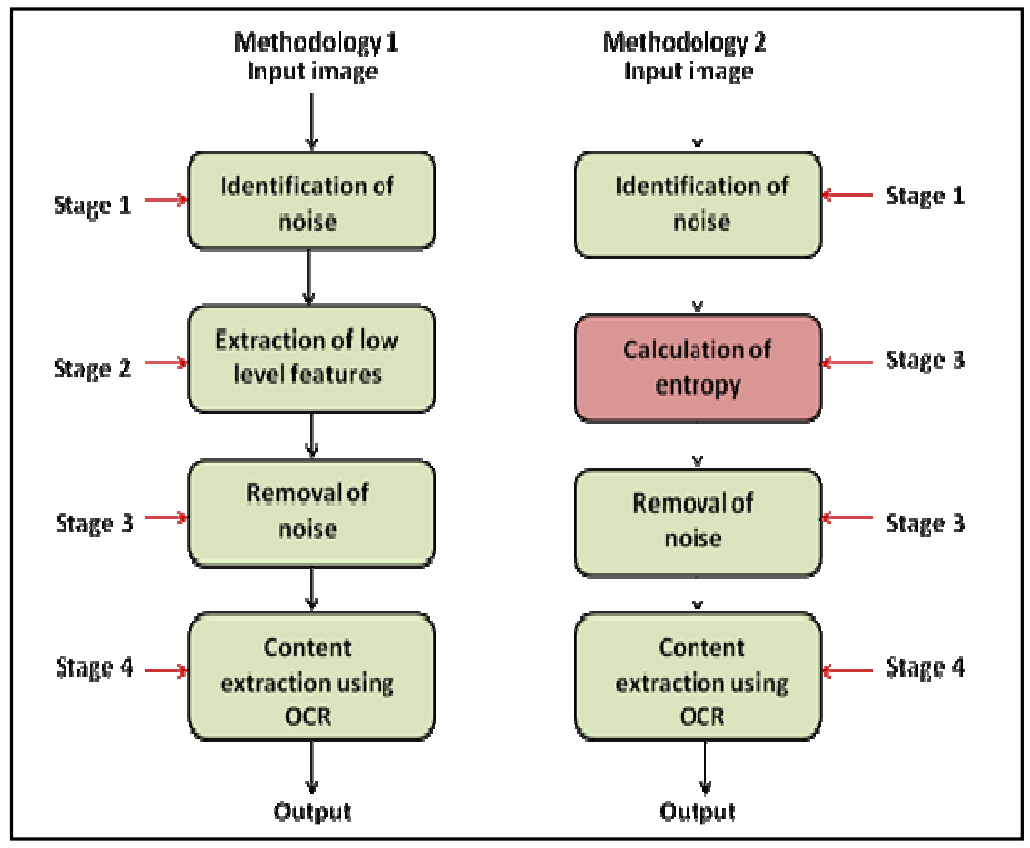

Figure 6. Basic framework of the two methodologies

\subsection{Methodology I}

Figure 7 shows the system architecture of methodology I.

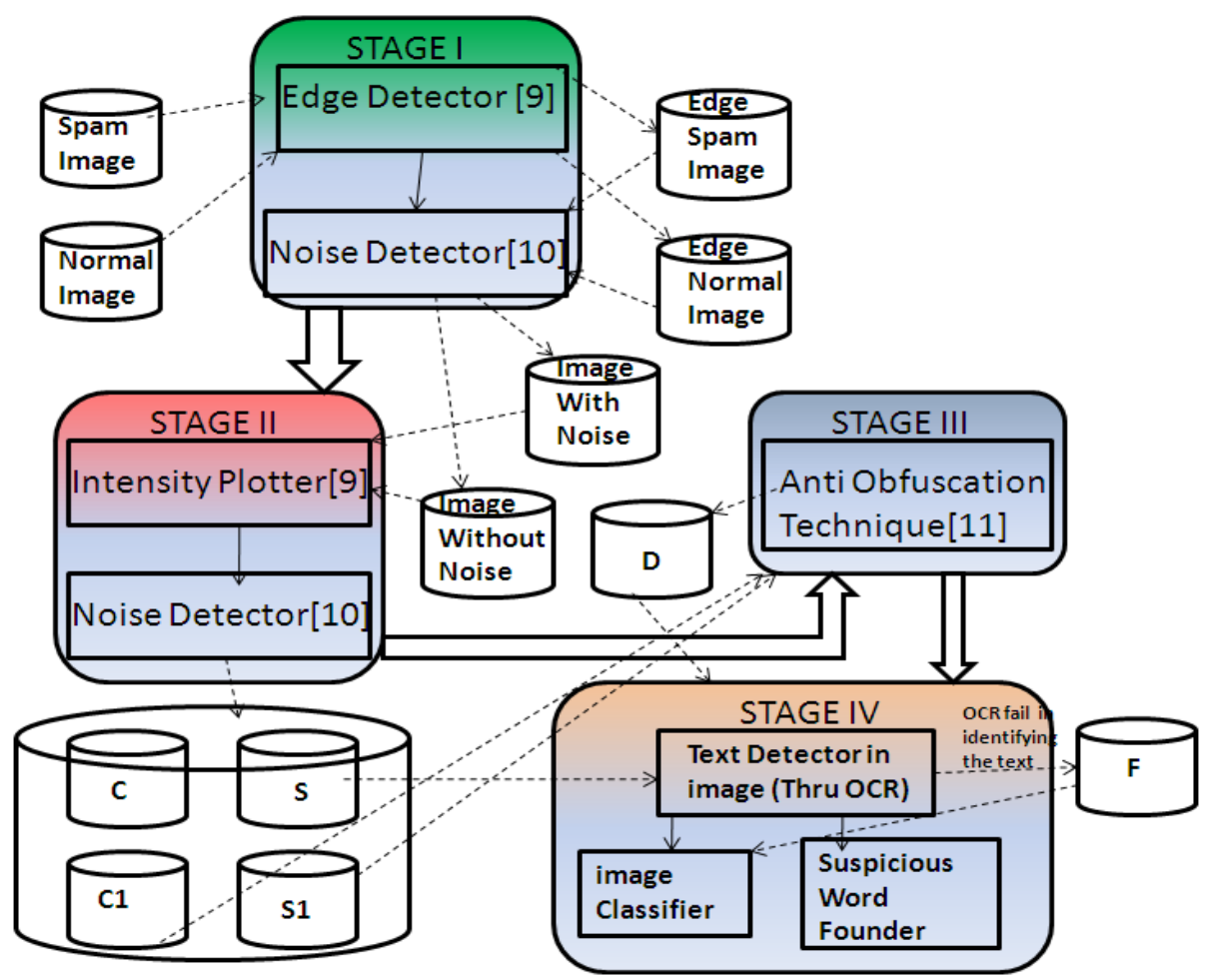

Figure 7. Methodology I 
International Journal of Network Security \& Its Applications (IJNSA), Vol.4, No.2, March 2012

\subsubsection{Stage I - Identification of noise}

Canny's edge detection [9] is used to identify noise in images. Images with noise are stored in set A and images without noise are stored in set B. Edge detection highlights even the slightest of noise added in an image. Images in Set A are more likely to be spam images. Set A is further classified into databases, namely -(A1) Dots \& Dashes (A2) Lines. This classification is done on the basis of type of noise usually found in spam images. Figure 9 shows a sample image for the noise found in Figure 8. They also display the difficulty to identify noise without edge detection.

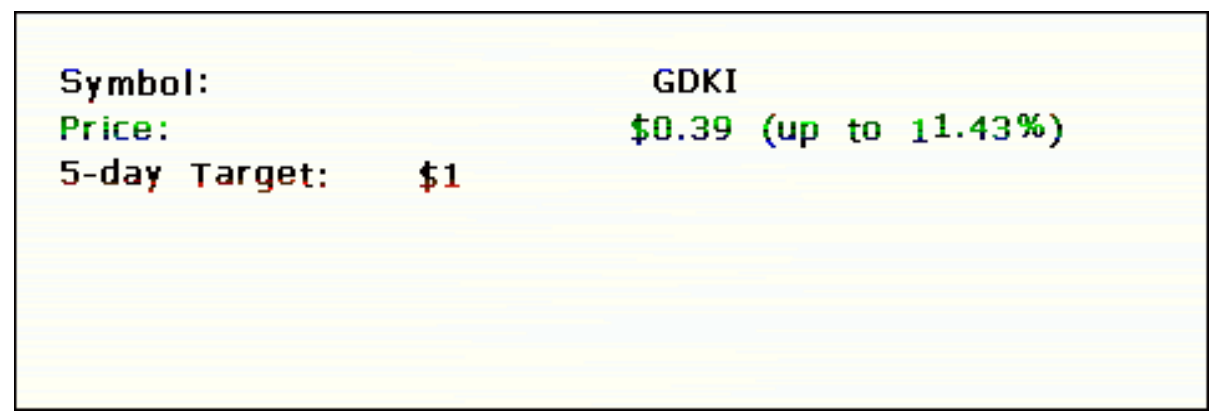

Figure 8. Image with noise

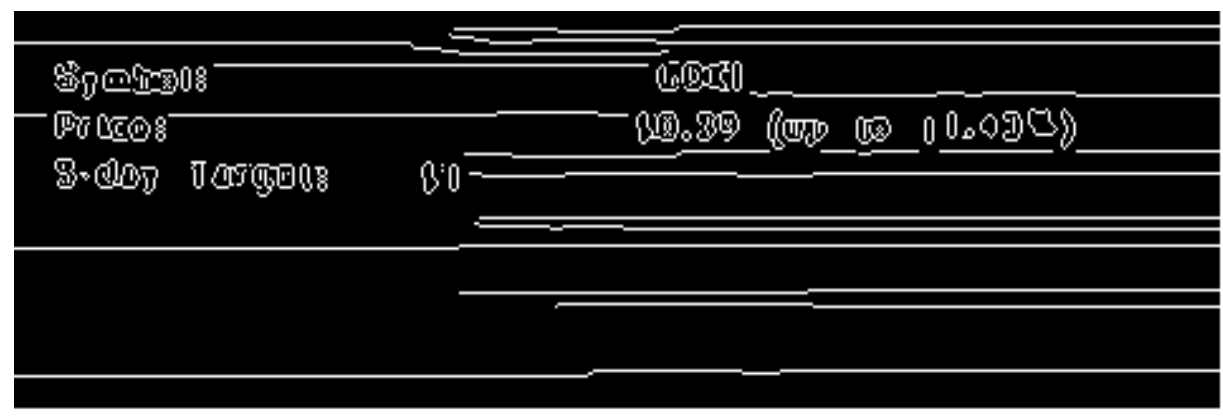

Figure 9. Edge detection shows line in fig 8

\subsubsection{Stage II - Extraction of low level features}

In this stage, all the input images pass through Intensity Plotter [9], which plots the variation of intensity along a line segment or a multiline path of an image. Since spam images are artificially generated, we expect their low level features to be different from those of images typically included as attachments to personal e-mails. The plots thus obtained do not change on varying the format of the image (from gif to jpeg or vice versa). Stage II has two sub stages. Images with noise are classified into two sets, $\mathrm{S}$ and $\mathrm{C}$. The classification is based on the difference in the shape of the plots obtained. Figures 10 and 11 show the intensity plots of normal and spam images respectively. Herein $\mathrm{X}$ and $\mathrm{Y}$ are two element vectors specifying $\mathrm{X}$ and $\mathrm{Y}$ data of the image. Images common to both Set B and Set C are labelled as normal images and are not processed further. Images in set $\mathrm{S}$ are directly passed to stage IV. Images with noise are classified (as mentioned above) on the basis of plots obtained in two sets, S1 and C1. Images in set $\mathrm{S} 1$ and $\mathrm{C} 1$ are passed to stage III. 


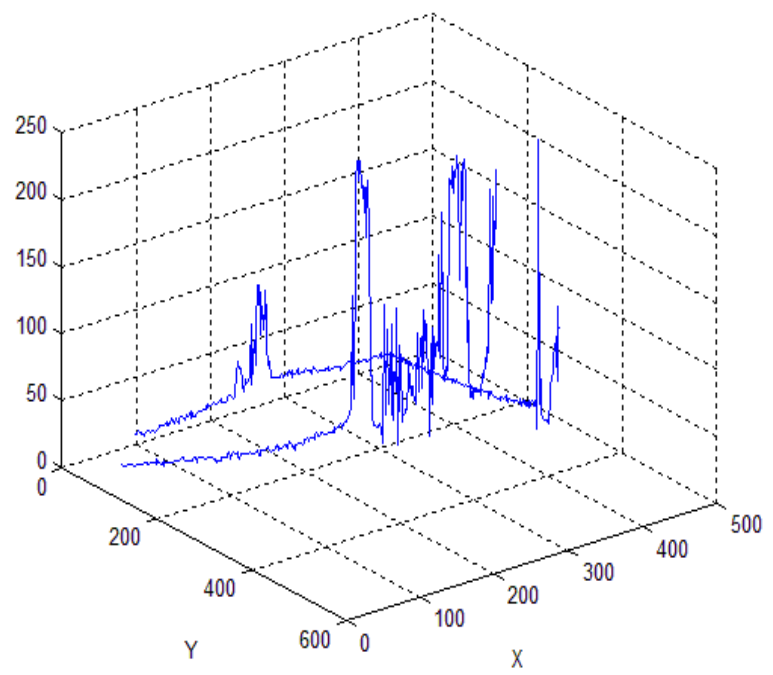

Figure 10. Intensity plot for normal images

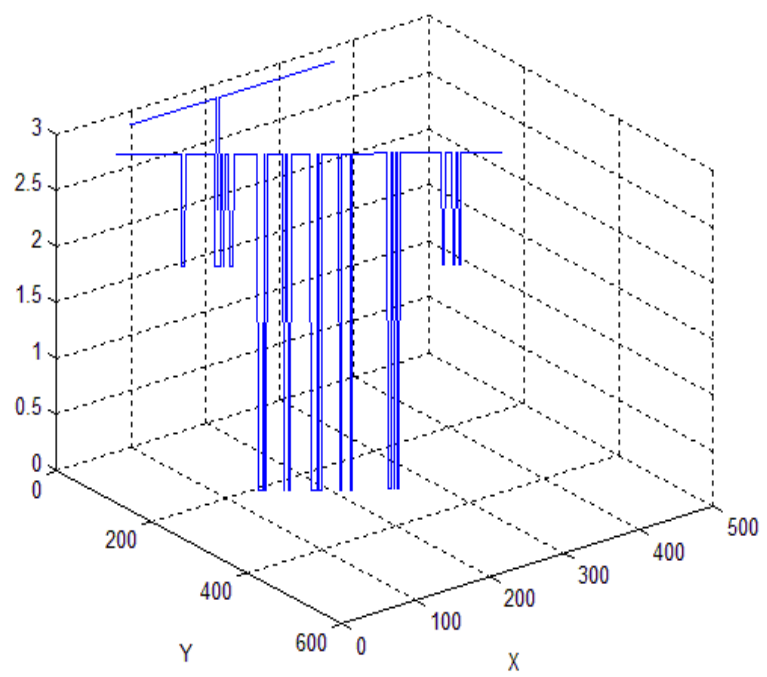

Figure 11. Intensity plot for spam images

\subsubsection{Stage III - Removal of noise}

Spammers add noise in images so that it becomes difficult for OCR to read the embedded text. To overcome this difficulty, obfuscation techniques are applied. Therefore, only images with noise are passed through this stage. In this technique we alter the RGB properties of the images. Images thus obtained are stored in set D. Figures 13 and 14 show a comparison between the text identified by OCR in Figure 12 before and after stage III. Table 1 shows a comparison between the words identified by OCR before and after stage III. 
International Journal of Network Security \& Its Applications (IJNSA), Vol.4, No.2, March 2012

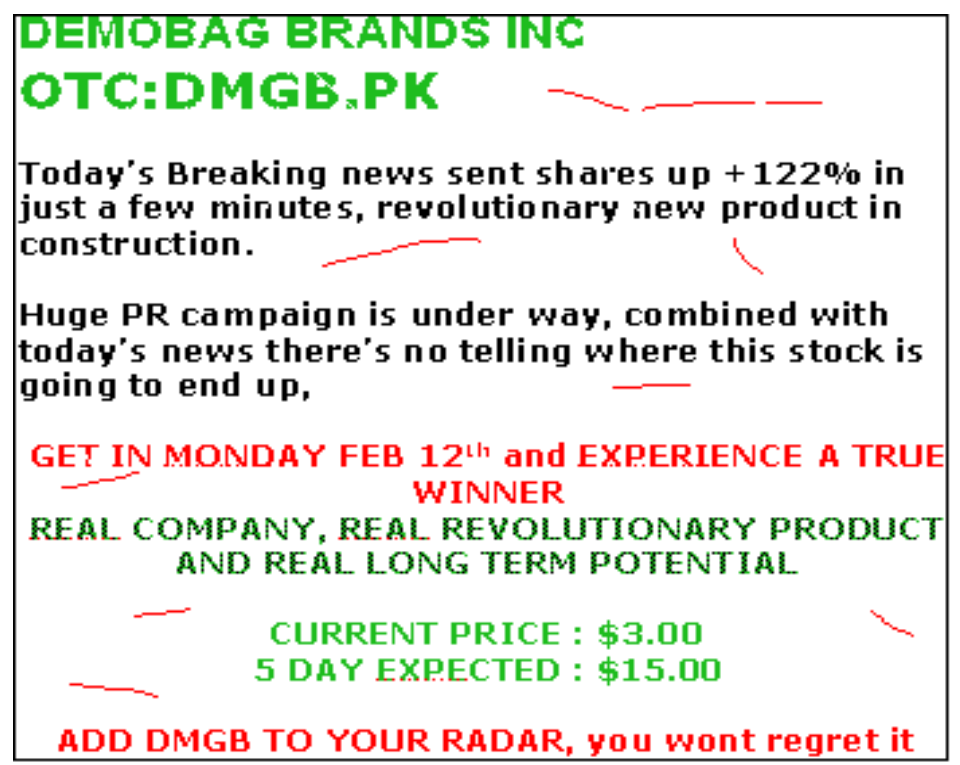

Figure 12. Original Image

\begin{tabular}{|c|}
\hline 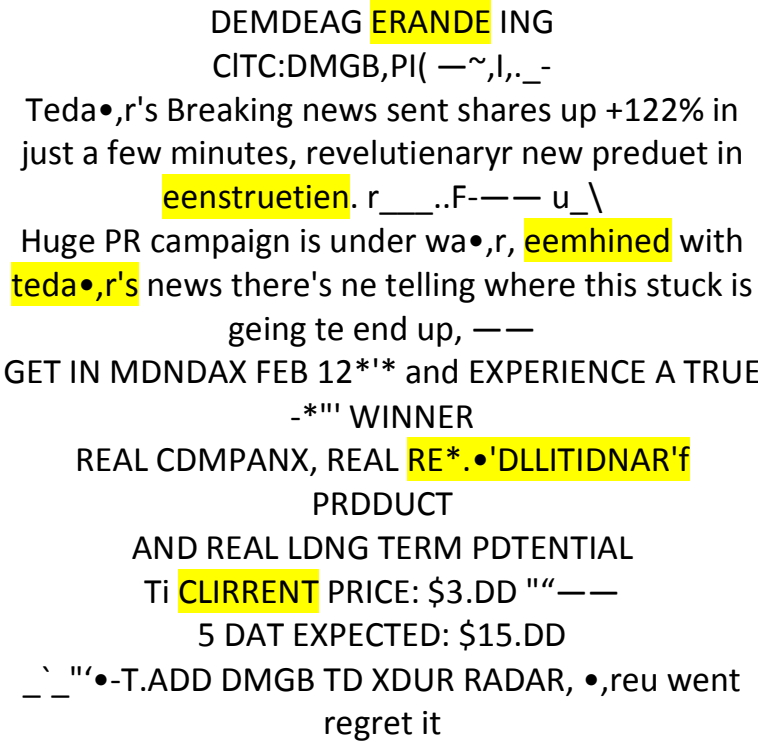 \\
\hline
\end{tabular}

Figure 13. Text identified in Figure 12 before applying anti-obfuscation technique 
International Journal of Network Security \& Its Applications (IJNSA), Vol.4, No.2, March 2012

DEMOBAG BRANDS INC
OTC:DMGB.PK
Today's Breaking news sent shares up $+122 \%$ in
Just a few minutes, revolutionary new product in
construction.
Huge PR campaign is under way, combined with
today's news there's no telling where this stock is
going to end up,
GET I,N MO.NDAY FEB 12 W and EXPERIENCE A TRUE
WINNER
REAL COMPANY, REAL REVOLUTIONARY PRODUCT
AND REALLONG TERMPOTENTIAL
CURRENT PRICE : \$3.00
5 DAY EXPECTED : \$15.00

Figure 14. Text identified in Figure 12 after applying anti-obfuscation technique

Table 1. Comparison between the words identified by OCR before and after applying stage III on Figure 12

\begin{tabular}{|c|c|c|}
\hline $\begin{array}{c}\text { Word in image with } \\
\text { noise }\end{array}$ & $\begin{array}{c}\text { Words as read by OCR } \\
\text { before stage III }\end{array}$ & $\begin{array}{c}\text { Words as read by } \\
\text { OCR after stage III }\end{array}$ \\
\hline DEMOBAG & DEMDEAG & DEMOBAG \\
\hline OTC:DMGB.PK & C1TC:DMGB,PIC--,I,- & OTC:DMGB.PK \\
\hline Today's & Teda.,r's & Today's \\
\hline Revolutionary & Revelutienaryr & revolutionary \\
\hline Construction & Eenstruction & construction \\
\hline Product & Preduet & product \\
\hline Blank & R-----f-----u- & going \\
\hline Going & Geing & way \\
\hline Way & Wa.r & stock \\
\hline Stock & stuck & to \\
\hline To & te & MO.NDAY \\
\hline MONDAY & MDNDAX & \\
\hline
\end{tabular}

\subsubsection{Stage IV - Content Extraction using OCR}

Input to this stage comprises of images in set $\mathrm{S}$ and D. Images are passed through OCR, which identifies embedded text and compares it with a list of keywords. If text identified matches with the list of spam words, then the image is labelled as spam image else it is a normal image. If OCR fails, then the graphs plotted in stage II are considered. Images in sets $\mathrm{S} 1$ and $\mathrm{S}$ are labelled as spam images. Images in set $\mathrm{C} 1$ are labelled as normal images. 


\subsection{Methodology II}

In this methodology a score system is employed in which scores are allotted to each image on the basis of their results obtained after each stage. Images with a score of 2 or more are classified as spam images and the rest are classified as normal images.

\subsubsection{Stage I - Identification of noise}

Canny's edge detection [9] is used to identify noise in images. Images with noise are more likely to be spam images hence images with noise are allotted a score of 1 while images without noise are allotted a score of 0 .

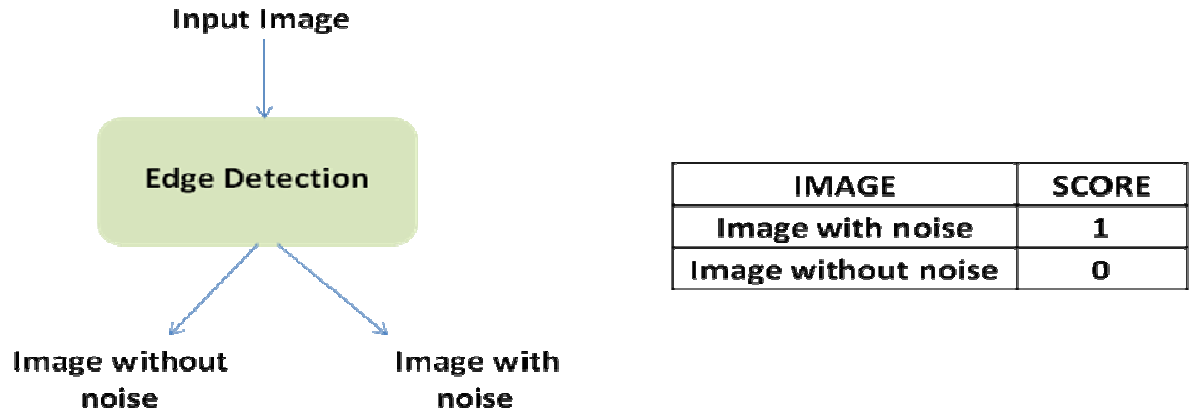

Figure 15. Stage I

\subsubsection{Stage II - Calculation of entropy}

In this stage, entropy of all the images is calculated. ENTROPY returns a scalar value representing the entropy of an intensity image. Entropy is a statistical measure of randomness that can be used to characterize the texture of the input image. Entropy is defined as $\operatorname{sum}\left(\mathrm{p} .{ }^{*} \log 2(\mathrm{p})\right)$ where $\mathrm{p}$ contains the histogram counts returned from IMHIST. In this stage score has been allotted to each image in accordance with the value of entropy and its extension. The basis for the 4 different categories has been explained in the experiments.

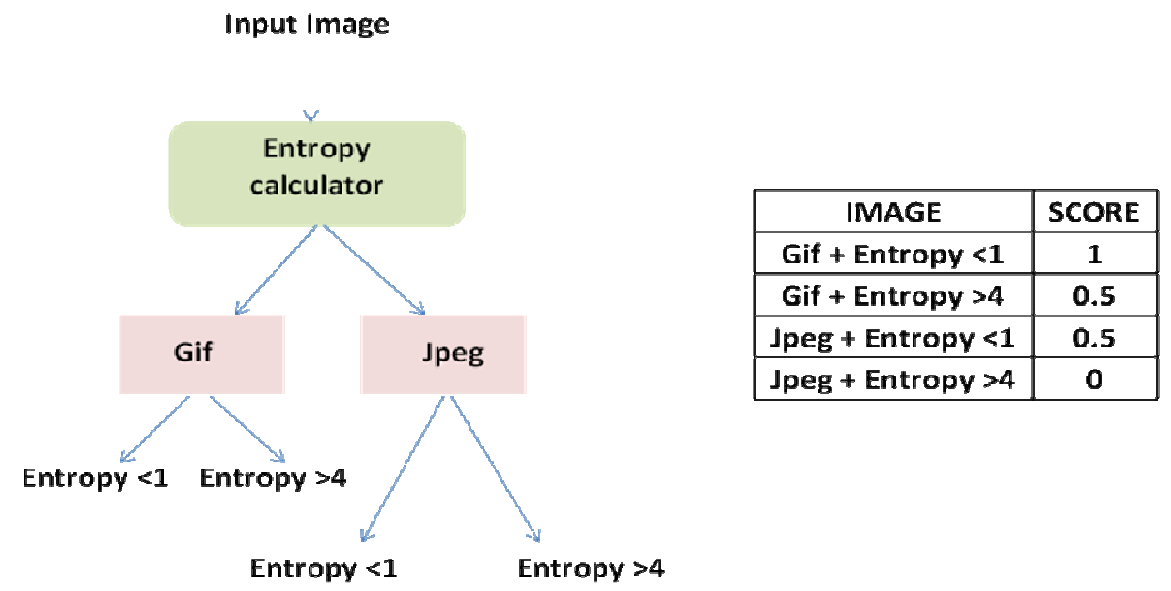

Figure 16. Stage II 
International Journal of Network Security \& Its Applications (IJNSA), Vol.4, No.2, March 2012

\subsubsection{Stage III - Removal of noise}

Only images with noise are passed through this stage. In this technique we alter the RGB properties of the images. Images thus obtained are further passed to stage IV.

\subsubsection{Stage IV - Content Extraction using OCR}

Images are passed through OCR, which identifies embedded text and compares it with a list of keywords. The images are allotted a score of 1 or 0 depending upon whether text identified matches or doesn't match with the list of words respectively.

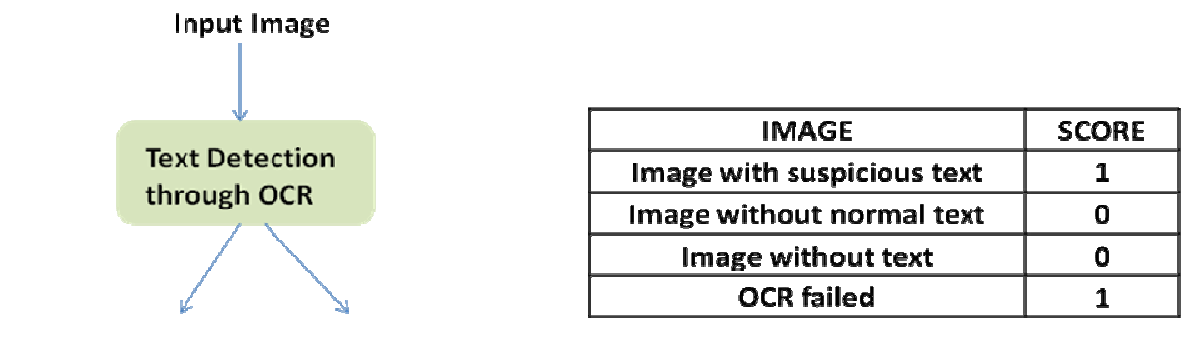

Suspicious text Normal text

Figure 17. Stage IV

\section{EXPERIMENTS AND RESULTS}

We have collected two sets of images to test the filter: spam images and normal images. The dataset consist of spam images in gif and jpeg format [12]. A set of 1984 images are taken, out of which 802 are normal images and 1182 are spam images. Experiments are performed on a system with the following specifications: 32 - bit operating system, $2.40 \mathrm{Ghz}$ processor and $4 \mathrm{~Gb}$ RAM. Matlab version 7.7 is used. It employs image processing techniques like Canny's edge detection, intensity plotter and entropy calculator. For applying anti obfuscation techniques, we make use of an online editor [11]. Free OCR V3 is used to extract the content of the spam image. The experiment has shown that our technique is effective in identifying spam images in any format. According to the experimental results, detection rate of methodology I is 0.92 , false positive rate is 0.0064 and false negative rate is 0.059 by calculation. Detection rate of methodology II is $93.3 \%$. Entropy of $96 \%$ of normal images is above 4 . The following are the results.

\subsection{Result I}

Figure 18. shows a comparison between the execution time of both the methodologies. Average execution time of methodology $\mathrm{I}$ is 0.174 seconds and the average execution time of methodology II is 0.06 seconds. However, methodology 2 is more time efficient than the first methodology but it is unable to give correct results when the extension of the image is changed. When we changed the extension of image from jpeg to gif we found out that the entropy falls by 3 units on an average thus decreasing its detection rate.

Intensity plotter on the other hand is totally independent of the extension of the image and hence does not show any such variation. 


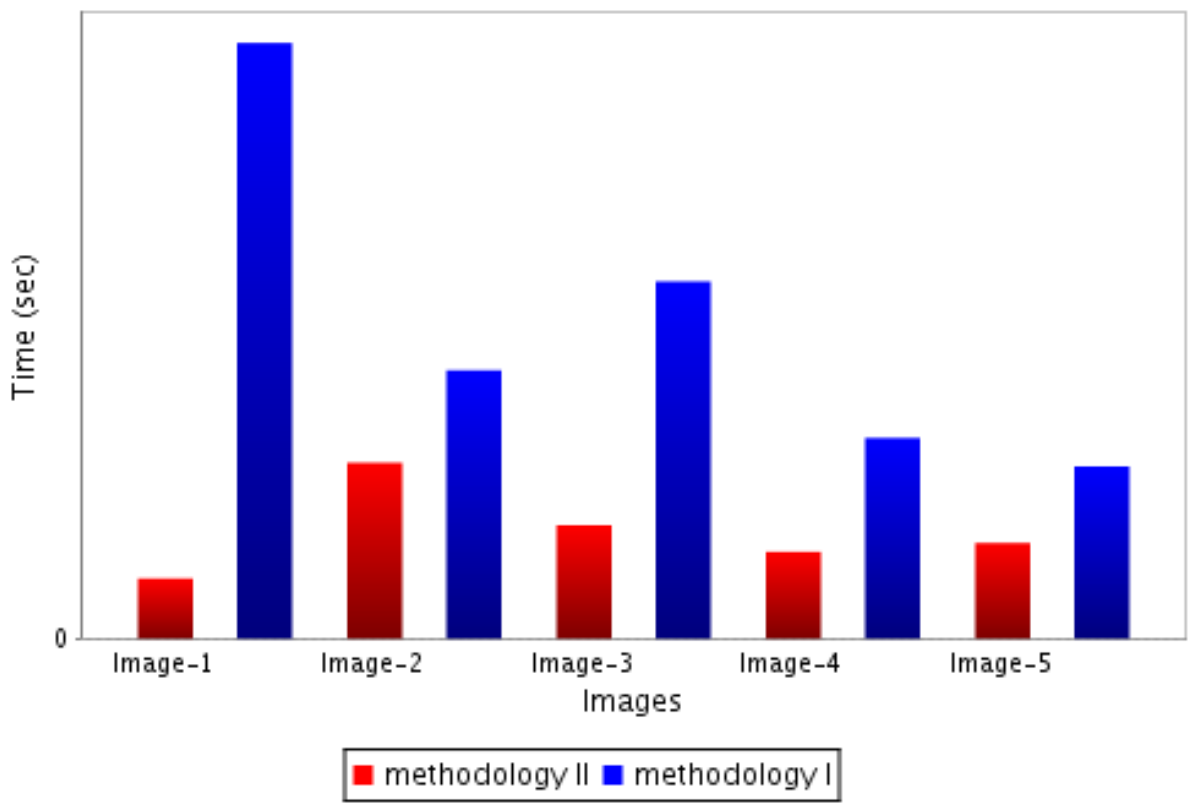

Figure 18. Comparison between execution time of methodology I and methodology II.

\subsection{Result II}

Figures 19 and 20 show the efficiency of spam and normal images which were correctly identified by using methodology I. We have tabulated the results by classifying hams and spam images into the following categories.

Spam Images: Advertisement, URL (Uniform Resource Locator), Pornography, Stock

Normal Images: Only Text, Text and Images, Only Images.

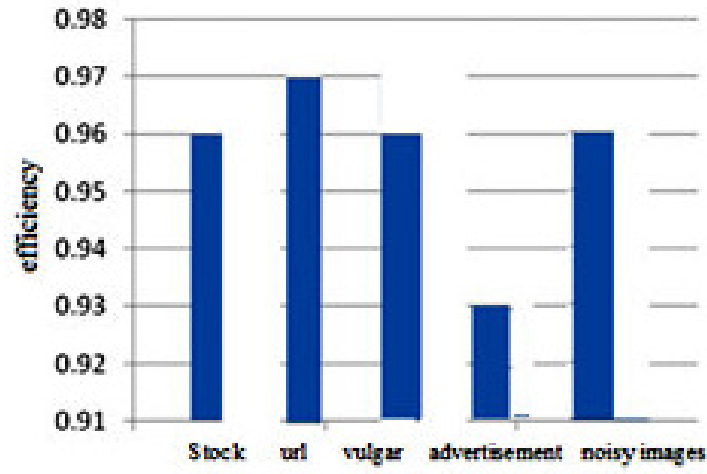

Figure 19. Histogram showing efficiency of spam images

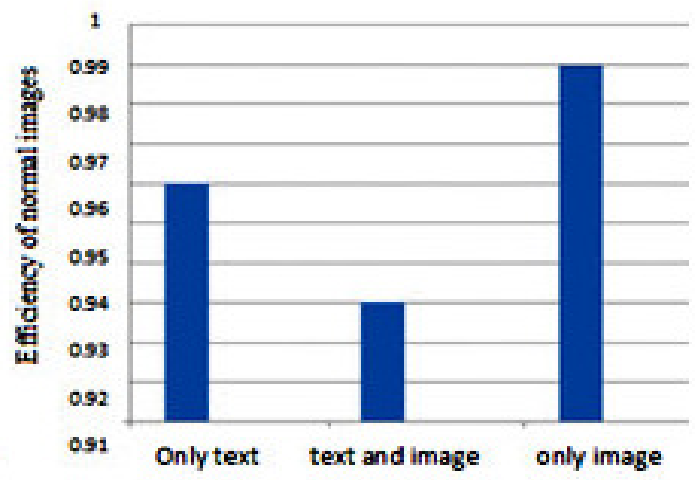

Figure 20. Histogram showing efficiency of normal images

\subsubsection{Discussion}

Hams with only images are easiest to identify because the range of colour components used is quite vast. Their intensity plots are curved and continuous. Their plots are easily distinguishable 
International Journal of Network Security \& Its Applications (IJNSA), Vol.4, No.2, March 2012

from those of spam images. Hams with only text consist mostly of survey forms, documents and newspaper articles. Due to the use of limited colours (mostly black and white) intensity plots of these images may be similar to that of spam images. Hence their efficiency is less than that of hams with only images. However hams with only text have efficiency higher than that of hams with both text and images because the former can be easily read by OCR . The presence of colourful images in the background of the latter makes it difficult for OCR to read the embedded text. Spam images concerning Advertisements are made attractive by adding colourful images and text so that they look like real advertisements. Hence, they are often confused with hams with both text and images and are toughest to identify.

\subsection{Result III}

We have taken five samples of a single image and increased its brightness from $20 \%$ to $80 \%$. Table 2 shows that OCR depends on the brightness of images. Tick $(\checkmark)$ shows images that OCR could read, cross $(x)$ shows images that OCR could not read and P shows images that OCR could partially read.

Table 2. Effectiveness of OCR on varying brightness of images

\begin{tabular}{|c|c|c|c|c|c|}
\hline Type of images & $\mathbf{2 0 \%}$ & $\mathbf{3 5 \%}$ & $\mathbf{5 0 \%}$ & $\mathbf{6 5 \%}$ & $\mathbf{8 0 \%}$ \\
\hline URL Spam & $\times$ & $\checkmark$ & $\checkmark$ & $\checkmark$ & $\times$ \\
\hline Stock Spam & $\times$ & $\checkmark$ & $\checkmark$ & $\mathrm{P}$ & $\times$ \\
\hline Vulgar Spam & $\times$ & $\checkmark$ & $\checkmark$ & $\mathrm{P}$ & $\times$ \\
\hline Advertisement Spam & $\times$ & $\checkmark$ & $\mathrm{P}$ & $\mathrm{P}$ & $\times$ \\
\hline Noise Spam & $\times$ & $\checkmark$ & $\checkmark$ & $\checkmark$ & $\times$ \\
\hline Ham with only text & $\checkmark$ & $\checkmark$ & $\checkmark$ & $\checkmark$ & $\checkmark$ \\
\hline
\end{tabular}

\subsubsection{Discussion}

OCR reads successfully the embedded text in an image if its brightness is confined to a particular range. However, it fails if brightness of an image deviates from a particular threshold having both upper and lower limit.

\subsection{Result IV}

We have varied the background colour of an image keeping its font colour constant. We have set the font colour at [R:255 G:0 B:0] and decreased the background colour from [R:255 G:240 $\mathrm{B}: 240]$ to [R:255 G:0 B:0] in sets of 30 keeping R constant. Figure 21 shows that the detection rate of OCR decreases as background colour approaches font colour. Black dots $(\bullet)$ show that OCR has failed. Red dots $(\bullet)$ show that OCR has been successful. The largest circle is [R:255 G:0 B:0]. 


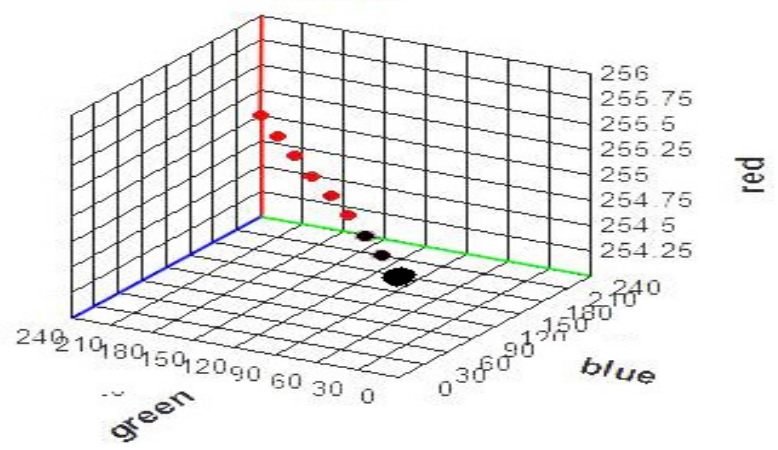

Figure 21. Detection rate of OCR decreases as background colour approaches font colour.

\subsubsection{Discussion}

OCR reads successfully the embedded text in an image if there exists a dissimilarity between font colour and background colour. As font colour approaches background colour it becomes difficult for OCR to read text.

\subsection{Result $V$}

We have artificially added 'salt \& pepper' noise in spam image, and have gradually increased the amount of noise added from 0.005 to 0.020 . On increasing noise, distortion in the graphs obtained by intensity plotter also increases. Also entropy of normal image decreases by $20 \%$ approximately as amount of noise added increases.

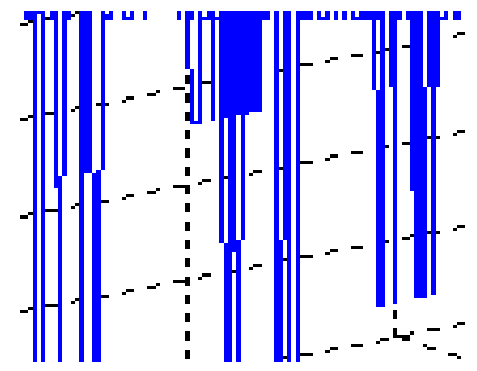

Figure 22. Intensity plot (noise is less)

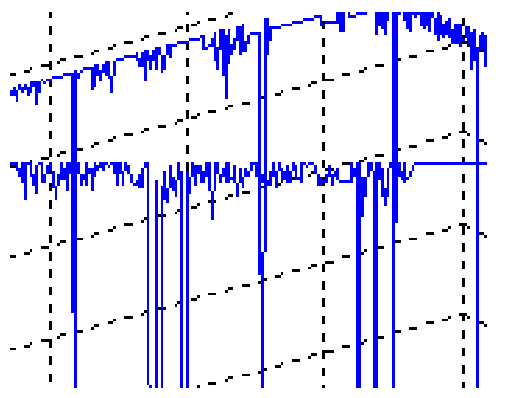

Figure 23. Intensity plot (noise is more)

\subsubsection{Discussion}

Intensity plot of artificial spam images tends to be straight. Intensity plotter gives correct plot for spam images till a particular amount of added noise, above that it gives a distorted plot for spam images. 
International Journal of Network Security \& Its Applications (IJNSA), Vol.4, No.2, March 2012

\section{CONCLUSION}

In this paper we present two methodologies for addressing image spam problems, however more methodologies can be proposed. It takes into account some of the recent evolutions of the spammers tricks in which obfuscation techniques are used to the extent that standard OCR tools become nearly ineffective. Methodology I extracts low level features while methodology II extracts metadata features of the image. Both the methodologies have their advantages and disadvantages. Experiments reveal that time taken by methodology II is less compared to methodology I, but entropy is not independent of the extension of image and shows a decreases in value when extension of the image changes from jpg to gif. However, intensity plotter is independent of extension and is effective in distinguishing between spam and normal images.

Anti-obfuscation techniques are applied to improve text filtering performance of the system. According to the experimental results, we have concluded that the detection rate depends on the type of spam images, i.e. whether it contains only text, text and images or images only. Spammers add noise and changes brightness of an image upto an extent only because then it hampers the clarity of the images and the user is unable to read the text.

\section{REFERENCES}

[1] G. Fumera, I. Pillai and F. Roli, "Spam Filtering Based On The Analysis Of Text Information Embedded Into Image”, Journal of Machine Learning Research (JMLR), vol. 7, pp. 2699-2720, December 2006.

[2] Apache.org (2011). The apache spamassassin project. Webpage (last accessed May 3, 2011): http://spamassassin.apache.org/index.html.

[3] M. Uemura and T. Tabata, "Design and Evaluation of a Bayesian-filter-based Image Spam Filtering Method", in Proceedings of the $2^{\text {nd }}$ International Conference on Information Security and Assurance (ISA '08), pp. 46-51, Busan, Korea, 24-26 April 2008.

[4] G. Yan, Y. Ming, Z. Xiaonan, B. Pardo, W. Ying, T. N. Pappas and A. Choudhary, ” Image Spam Hunter", in Proceeding of the International Conference on Acoustics, Speech and Signal Processing (ICASSP '08), pp. 1765-1768, Las Vegas, Nevada, USA, 30 March- 4 April 2008.

[5] C. Wang, F. Zhang, F. Li and Q. Liu, "Image Spam Classification based on Low Level Image Features", in Proceeding of the $8^{\text {th }}$ International Conference on Communications, Circuits and Systems (ICCCAS '10), pp. 290-293, Chengdu China, 28-30 July 2010.

[6] P. Klangpraphant, and P. Bhattarakosol, "PIMSI: A Partial Image Spam Inspector”, in Proceeding of the $5^{\text {th }}$ International Conference on Future Information Technology (FutureTech), pp. 1-6, Busan, South Korea, 21-23 May 2010.

[7] J. H. Hsia and M. S. Chen, "Language-Model-based Detection Cascade for Efficient Classification of Image-based Spam e-mail", in Proceeding of the International Conference on Multimedia and Expo (ICME '09), pp. 1182-1185, New York USA, 28 June-3 July 2009.

[8] M. Soranamageswari and C. Meena, "Statistical Feature Extraction for Classification of Image Spam Using Artificial Neural Networks", in Proceeding of the $2^{\text {nd }}$ International Conference on Machine Learning and Computing (ICMLC '10), pp. 101-105, Bangalore India, 9-11 February 2010.

[9] Mathworks The Matlab image processing toolbox.M, Available at http://www.mathworks.com/access/helpdesk/help/toolbox/images/.. downloaded on july 10

[10] Bag of Visual words Model: Recognizing Object Categories, Available at http://www.robots.ox.ac.uk/ az/icvss08_az_bow.pdf.

[11] Image editor, http://www.lunapic.com/editor/?action=contrast, downloaded on july 10,2011 
International Journal of Network Security \& Its Applications (IJNSA), Vol.4, No.2, March 2012

[12] Image spam dataset, http://www.cs.jhu.edu/ mdredze/datasets/image_spam/, downloaded on june 3,2011 .

[13] Bhaskar Mehta, Saurabh Nangia, Manish Gupta, "Detecting Image Spam using Visual Features and Near Duplicate Detection", in Proceeding of the International World Wide Web Conference Committee (IW3C2), April 21-25, 2008. 